Research Article | Internal Medicine

\title{
Dual-Energy Multidetector Computed Tomography: A Highly Accurate Non-Invasive Tool for in Vivo Determination of Chemical Composition of Renal Calculi
}

\author{
Shafqat Shabir Bhawani ${ }^{1 *}$, Majid Jehangir ${ }^{1}$, Mohammad Masood ${ }^{1}$, Sajjad Ahmad Dar ${ }^{1}$, \\ Sajad Nazir Syed²
}

\begin{abstract}
Introduction. Computed tomography is more accurate than excretory urography in evaluation of renal stones due to its high sensitivity and temporal resolution; it permits sub-millimetric evaluation of the size and site of calculi but cannot evaluate their chemical composition. Dual-energy computed tomography allows evaluating the chemical composition of urinary calculi using simultaneous image acquisition at two different energy levels. The objective of the research was to determine renal stone composition using dual-energy multidetector computed tomography, and its correlation with post-extraction chemical analysis of stones.

Materials and Methods. This prospective study was conducted in the Department of Radiodiagnosis and Imaging from September 2017 to March 2019. A total of 50 patients with urolithiasis at the age of 18-70 years were included in the study. Dual-energy computed tomography ratios of various stones were noted, and preoperative composition of calculi was given based on their colour and dual-energy computed tomography ratio. These results were compared with the post-extraction chemical analysis of stones (using Fourier infrared transform spectroscopy as the standard comparative method.)

Results. The most common type of calculi in our study population was calcium oxalate stones $(78 \%)$ followed by uric acid stones (12\%), cystine stones (6\%) and hydroxyapatite stones $(4 \%)$. The dual-energy ratio of calcium oxalate, uric acid, cystine and hydroxyapatite stones ranged from 1.38-1.59, 0.94-1.08, and 1.20-1.28 and $1.52-1.57$, respectively, with the mean dual-energy ratio of $1.43,1.01,1.25$ and 1.55 , respectively. Dual-energy computed tomography was found to be $100 \%$ sensitive and specific for differentiating uric acid stones from non-uric acid stones. The sensitivity and specificity in differentiating calcium oxalate calculus from non-calcium oxalate calculus was $97.5 \%$ and $90.9 \%$, respectively, with $96 \%$ accuracy and kappa value of 0.883 suggesting strong agreement.

Conclusions. Dual-energy computed tomography is highly sensitive and accurate in distinguishing between various types of renal calculi. It has vital role in management as uric acid calculi are amenable to drug treatment, while most of non-uric acid calculi require surgical intervention.

\section{Keywords}

Dual-Energy Computed Tomography; Calculi; Dual-Energy Ratio; Composition; Peak Kilovoltage; Uric Acid; Hounsfield Unit

${ }^{1}$ Department of Radiodiagnosis and Imaging, Government Medical College, Srinagar, Jammu and Kashmir, India

${ }^{2}$ Department of Urology, Government Medical College, Srinagar, Jammu and Kashmir, India

*Corresponding author: shafqat.shabir0081@gmail.com
\end{abstract}




\section{Introduction}

Nephrolithiasis is a major cause of morbidity worldwide. The incidence of renal stone disease is high, with a lifetime risk of $6 \%$ in women and $12 \%$ in men [1-3]. According to the recent epidemiological data, the prevalence and incidence rates of urolithiasis have increased considerably in nearly all countries [4]. Most renal stones (75-80\%) are calciumcontaining stones followed by struvite stones (10-15\%), uric acid stones (6\%) and cystine stones (1-2\%) [5]. Clinical presentations of urinary stone disease include renal colic or atypical abdominal pain, gross haematuria, urinary urgency, nausea, and vomiting [6]. Earlier, plain abdominal X-rays and intravenous urograms were the main modalities used for the diagnosis of renal stones, but recently non-enhanced conventional single-energy computed tomography (CT) has become the imaging modality due to its high sensitivity and specificity [7]; however, the major drawback of this technique is that it cannot reliably predict calculus composition [8]. Dualenergy CT (DECT) gives information regarding the chemical characterization of renal stones in addition to the assessment of stone size, location, and surface [9]. Preoperative determination of the stone subtype helps the clinician better decide treatment options for the patient as uric acid calculi are amenable to drug treatment and may not require surgery [10]. Due to high rates of recurrent stone disease, preoperative characterization of stone type plays vital role in management.

The objective of this research was to determine the level of agreement between DECT and post extraction chemical composition of calculi in the differentiation of uric acid calculi from non-uric acid calculi and calcium oxalate calculi from non-calcium oxalate calculi.

\section{Materials and Methods}

This prospective study was conducted in the Department of Radiodiagnosis and Imaging in a study period of 18 months from September 2017 to March 2019. A total of 50 patients (30 males, 20 females) were included in the study.

\section{Inclusion Criteria}

Patients (both males and females) with urolithiasis admitted to the Department of Urology and referred to the Department of Radiodiagnosis and Imaging for DECT were included in the study.

\section{Exclusion Criteria}

Pregnancy was the only exclusion criterion.

\section{Methodology}

The patients were subjected to non-contrast DECT of the abdomen and pelvis with interest areas including kidneys, ureter, and bladder. Various features of detected stone were noted, including size, location, colour, volume, Hounsfield units (HU) values at 100/140 $\mathrm{kVp}$ levels, and dual-energy ratios (DE ratios) to determine the stone composition using a fourth generation 256-slice dual-source dual-energy CT scanner (SOMATOM Definition Flash, Siemens Healthcare, Forchheim, Germany). Images were acquired at slice thickness of $5 \mathrm{~mm}$. Images were reconstructed to produce 3-mm-thick slice sections. All exams were visualized on the axial, coronal, and sagittal planes. Images acquired with DECT were processed using a dedicated Siemens workstation. The various steps in detail are as under:

\section{Image Acquisition}

DE scan was performed using 256-slice dual-source dualenergy scanner which acquires the images at $100 / 140 \mathrm{kVp}$ in two different planes by the two X-ray tubes (Master tube and slave tube) angled at $90^{\circ}$ to each other.

\section{Post-Processing and Image Interpretation}

The ability of DECT to differentiate two materials depends on the characteristic DE ratio. The difference between the DE number ratios for any two materials is determined by the separation between the low and high energy spectra and the effective atomic numbers of the materials [11].

$D E$ Ratio $=H U$ lower $k V P / H U$ higher $k V P$

The stone marker was placed on the desired stone which revealed various DE parameters of the stone like $\mathrm{HU}$ values at 100/140/mixed image, stone volume, stone size and the most important DE ratio besides giving it a colour for classifying into uric acid (red colour) and non-uric acid (blue colour) stone. The DE ratio was, then, used to classify the stone into uric acid, calcium oxalate, cystine and hydroxyapatite calculi.

\section{Post-Extraction Analysis Comparison}

Post-extraction chemical analysis of the stones for their composition was conducted by Fourier Transform Infrared Spectroscopy (FTIR) which was used as control for the confirmation of stone composition by dual-source DECT.

\section{Statistical Methods}

The recorded data were compiled and entered in a spreadsheet (Microsoft Excel) and then, exported to data editor of SPSS- Statistical package for the social sciences Version 20.0 (SPSS Inc., Chicago, Illinois, USA). Continuous variables were expressed as Mean $\pm \mathrm{SD}$ and categorical variables were summarized as frequencies and percentages. Sensitivity, specificity, likelihood ratio, positive and negative predictive values were used to determine the diagnostic accuracy of DECT against post-extraction analysis. Diagnostic accuracy of DECT in differentiating different types of calculi was assessed using Cohen's kappa agreement. Kappa value of greater than 0.75 represents excellent agreement beyond chance. A p-value of less than 0.05 was considered statistically significant.

\section{Results and Discussion}

This prospective study included 50 patients with nephrolithiasis to explore the reliability of DECT in determining the chemical composition of urinary calculi. The most affected age 
group included patients at the age of 30-44 years (18 cases) representing $36 \%$ of cases. Males were more affected than females, with a male to female ratio of 3:2.

The most common type of calculi in our study population was calcium oxalate stones (39 calculi - $78 \%$ of cases) followed by uric acid stones ( 6 calculi - $12 \%$ of cases), cystine stones ( 3 calculi - $6 \%$ of cases) and hydroxyapatite stones ( 2 calculi - $4 \%$ of cases). The results of our study were in agreement with the study conducted by Mangalvati G et al. [12] and Basha $\mathrm{M}$ et al. [13]. Table 1 shows age- and gender-wise distribution of various renal stones encountered in the study. In our study, 28 (56\%) patients had renal colic as the pre-

Table 1. Age- and gender-wise distribution of various types of stones.

\begin{tabular}{lcccccccc}
\hline & M & F & M & F & M & F & M & F \\
\hline$<30$ & 4 & 8 & 0 & 0 & 0 & 0 & 0 & 0 \\
$30-44$ & 12 & 2 & 1 & 0 & 2 & 0 & 1 & 0 \\
$45-59$ & 4 & 5 & 1 & 2 & 0 & 1 & 0 & 0 \\
$60-74$ & 4 & 0 & 0 & 2 & 0 & 0 & 1 & 0 \\
Total & 24 & 15 & 2 & 4 & 2 & 1 & 2 & 0 \\
\hline
\end{tabular}

Notes: M - Males, F - Females.

dominant symptom; 4 (8\%) patients presented with nausea and vomiting; 2 (4\%) patients suffered from fever; $16(32 \%)$ patients were diagnosed with haematuria.

Most of the calculi were in the renal calyceal system $(40 \%)$ followed by the ureters $(36 \%)$ and the renal pelvis (24\%). Calcium oxalate calculi (46\%) were predominant in the renal calyceal system, while all the uric acid calculi were found in the ureter (Fig. 1).

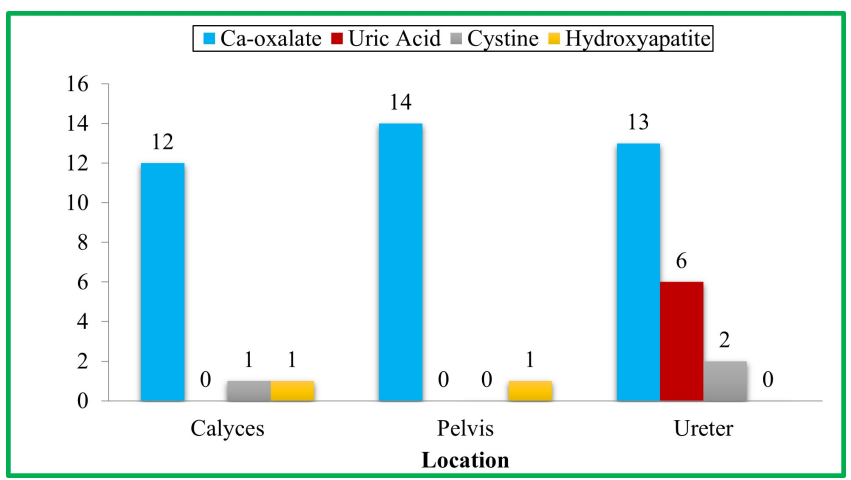

Figure 1. Bar diagram showing the distribution of various calculi based on the location.

The mean volume of calcium oxalate calculi was $1,306 \mathrm{~mm}^{3}$; the mean volume of hydroxyapatite stones was $1,594 \mathrm{~mm}^{3}$ (the highest one); the mean volume of uric acid calculi was $352 \mathrm{~mm}^{3}$; the mean volume of cystine calculi was $152 \mathrm{~mm}^{3}$ (the lowest one.)

Mean attenuation values and range of different types of stones at 100/140 kVp and mixed $\mathrm{kVp}$ are shown in Table 2.
Table 2. Mean attenuation values and range of different types of stones at 100/140 $\mathrm{kVp}$ and mixed $\mathrm{kVp}$.

\begin{tabular}{lccc}
\hline \multirow{2}{*}{$\begin{array}{l}\text { Type } \\
\text { of stone }\end{array}$} & \multicolumn{3}{c}{ Mean HU values at: } \\
\cline { 2 - 4 } & $\begin{array}{c}100 \mathrm{kVp} \\
\text { (range) }\end{array}$ & $\begin{array}{c}140 \mathrm{kVp} \\
\text { (range) }\end{array}$ & $\begin{array}{c}\text { mixed kVp } \\
\text { (range) }\end{array}$ \\
\hline Calcium & 1296 & 909 & 1104 \\
Oxalate & $(502-1708)$ & $(356-1197)$ & $(427-1467)$ \\
Uric Acid & 557 & 553 & 555 \\
& $(431-625)$ & $(439-603)$ & $(432-601)$ \\
Cystine & 480 & 417 & 437 \\
Hydroxy- & $(236-941)$ & $(204-784)$ & $(210-865)$ \\
apatite & 1210 & 787 & 1022 \\
\hline
\end{tabular}

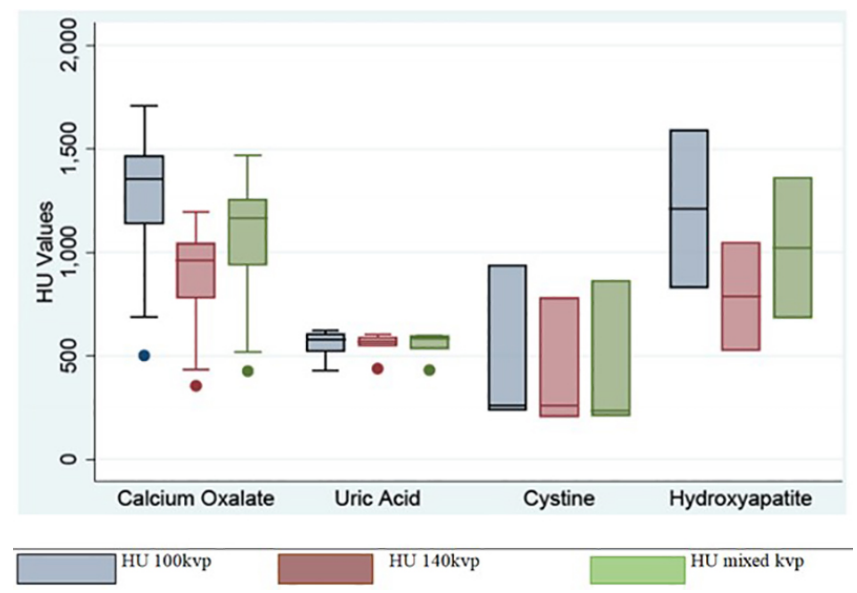

Figure 2. Box plot chart depicting the relationship between $\mathrm{HU}$ values (at $100 \mathrm{kVp}, 140 \mathrm{kVp}$ and mixed $\mathrm{kVp}$ ) and the type of calculus.

A box plot chart depicting the relationship between $\mathrm{HU}$ values (at $100 \mathrm{kVp}, 140 \mathrm{kVp}$ and mixed $\mathrm{kVp}$ ) and the type of calculus is shown in Fig. 2. The DE ratio of calcium oxalate, uric acid, cystine and hydroxyapatite stones ranged from 1.38-1.59, $0.94-1.08$, and 1.20-1.28 and 1.52-1.57, respectively, with the mean DE ratio of $1.43,1.01,1.25$ and 1.55 , respectively (Fig. 3). In our study, the mean DE ratio of uric acid stones $(1.01 \pm 0.061)$ was different from the mean DE ratio of nonuric acid stones $(1.42 \pm 0.162)$, with p-value $<0.001$. These findings agreed with the study done by Hidas G et al. [7], who found that the DE ratio of less than 1.1 was typical of uric acid calculi, the DE ratio of 1.1-1.24 was typical of cystine calculi and the DE ratio greater than 1.24 indicated calcium calculi. The sensitivity and specificity for differentiating calcium oxalate calculi from non-calcium oxalate calculi was found to be 97.5\% and $90.9 \%$, respectively, with $96 \%$ accuracy and a high positive likelihood ratio of 10.72 (Table 3 ). The sensitivity and specificity of differentiating uric acid calculi from non-uric acid calculi was $100 \%$ (Table 3) which agreed with the study done by Ilyas M et al. [14] and Stolzman P et al. [15]. The 
Table 3. Diagnostic accuracy of DECT against post-extraction analysis.

\begin{tabular}{|c|c|c|c|c|}
\hline & \multicolumn{2}{|c|}{$\begin{array}{c}\text { Calcium oxalate calculi } \\
\text { vs } \\
\text { non-calcium oxalate calculi }\end{array}$} & \multicolumn{2}{|c|}{$\begin{array}{c}\text { Uric acid calculi } \\
\text { vs } \\
\text { non-uric acid calculi }\end{array}$} \\
\hline & Value & $95 \% \mathrm{CI}$ & Value & $95 \% \mathrm{CI}$ \\
\hline Sensitivity & 97.5 & $86.8-99.9$ & 100 & $54.1-100$ \\
\hline Specificity & 90.9 & $58.7-99.8$ & 100 & $91.9-100$ \\
\hline Positive predictive value & 97.5 & $86.8-99.9$ & 100 & $54.1-100$ \\
\hline Negative predictive value & 90.9 & $58.7-99.8$ & 100 & $91.9-100$ \\
\hline
\end{tabular}

Notes: CI - confidence interval.

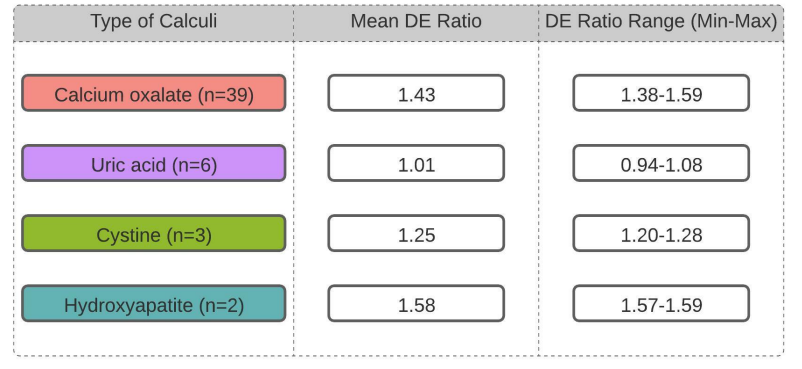

A

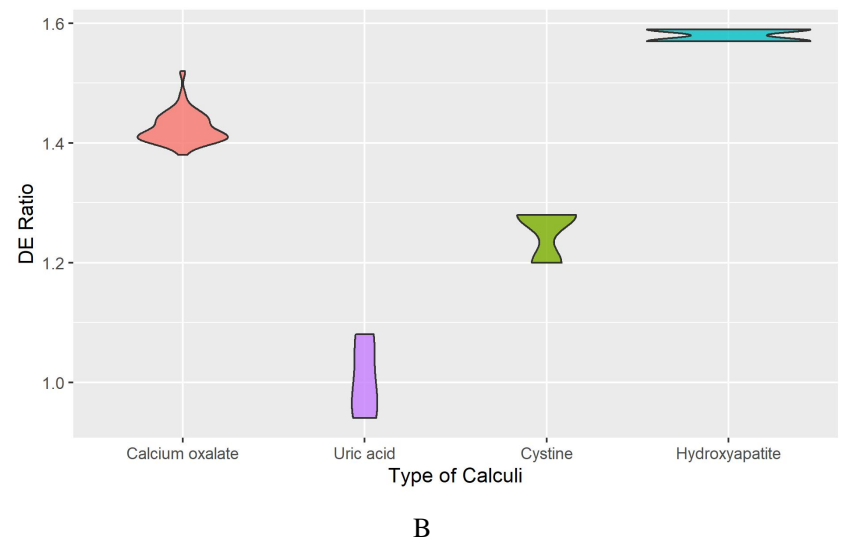

Figure 3. Diagram (A) and violin plot (B) depicting the mean $\mathrm{DE}$ ratio and the $\mathrm{DE}$ ratio range of calculi.

graphical representation of the DE ratios of calcium oxalate stones vs non-calcium oxalate stones and uric acid calculi vs non-uric acid calculi are depicted in Fig. 4 (A, B).

Fig. 5 shows post-processed DECT images with colour coding of various renal calculi.

In our study, the calculus composition was correctly assessed in 48 out of 50 (96\% accuracy) patients. Two calculi were misclassified with DECT: one of the calculi was in the renal pelvis of an obese male (calcium oxalate by DECT with the DE ratio of 1.52, chemical composition was determined as hydroxyapatite) and second calculus was small (hydroxyapatite by DECT with the DE ratio of 1.57 , chemical composition was determined as calcium oxalate). These find-

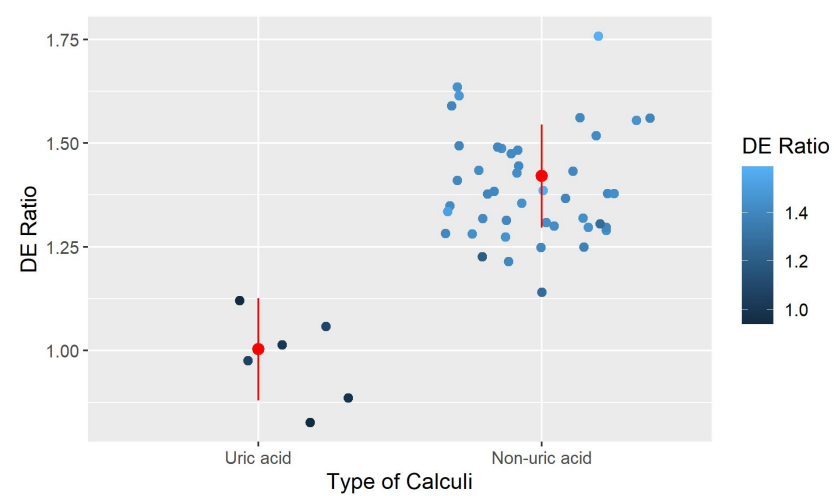

A

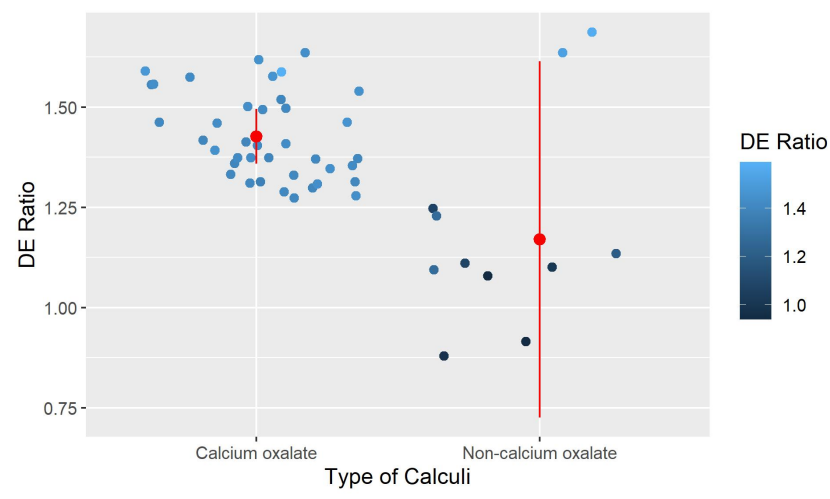

B

Figure 4. Scatter plot displaying the comparative DE ratios of non-uric acid calculi vs uric acid calculi (A), calcium oxalate calculi vs non-calcium oxalate calculi (B).

ings agreed with the observations of Thomas C et al. [16] who found that DECT was less accurate in characterizing the chemical composition of urinary calculi in obese patients.

Our study showed perfect agreement between DECT and chemical analysis in differentiating uric acid calculi and nonuric acid calculi $(\mathrm{p}<0.001$ and kappa value $=1.0)$. Thus, DECT is just as sensitive and accurate as chemical analysis in distinguishing uric acid calculi from non-uric acid calculi. Significant agreement exists between DECT and chemical analyses in terms of differentiating calcium oxalate 


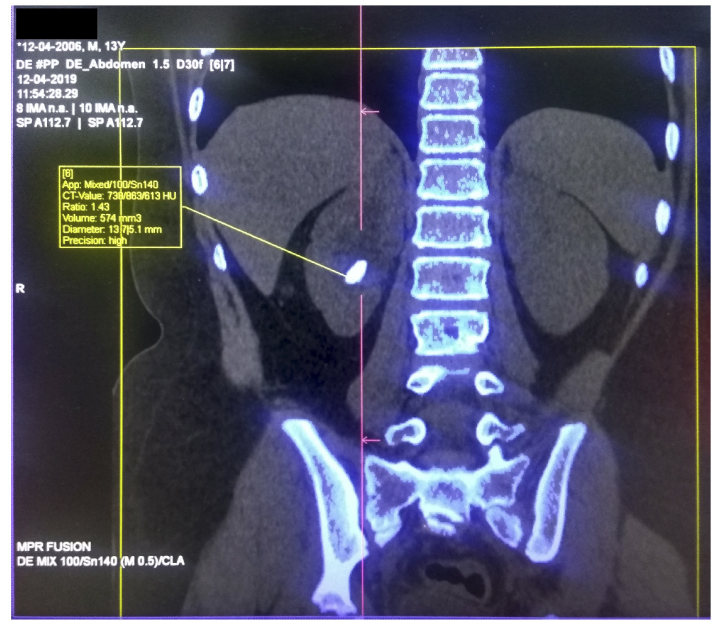

A

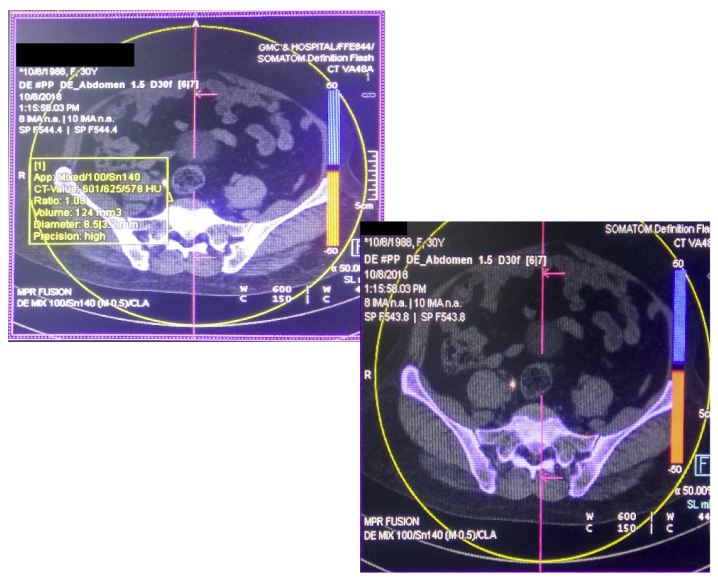

C

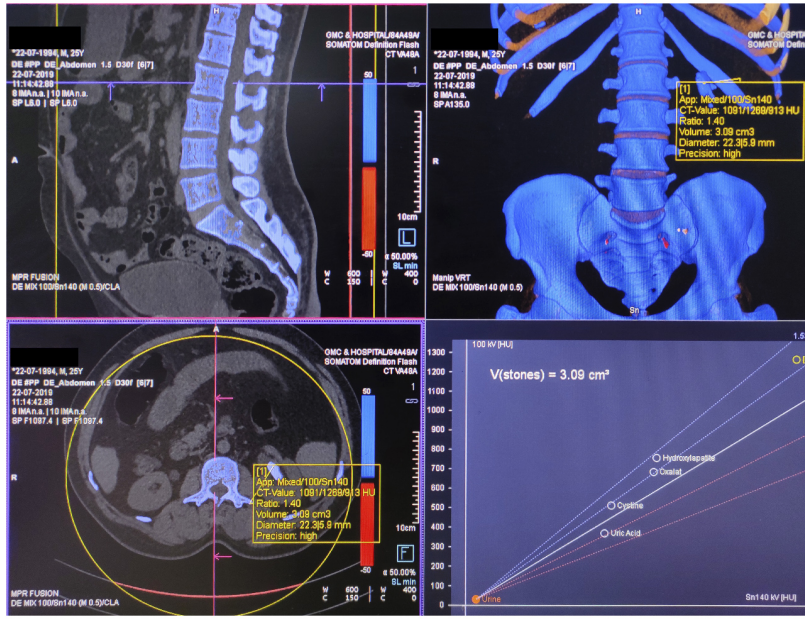

B

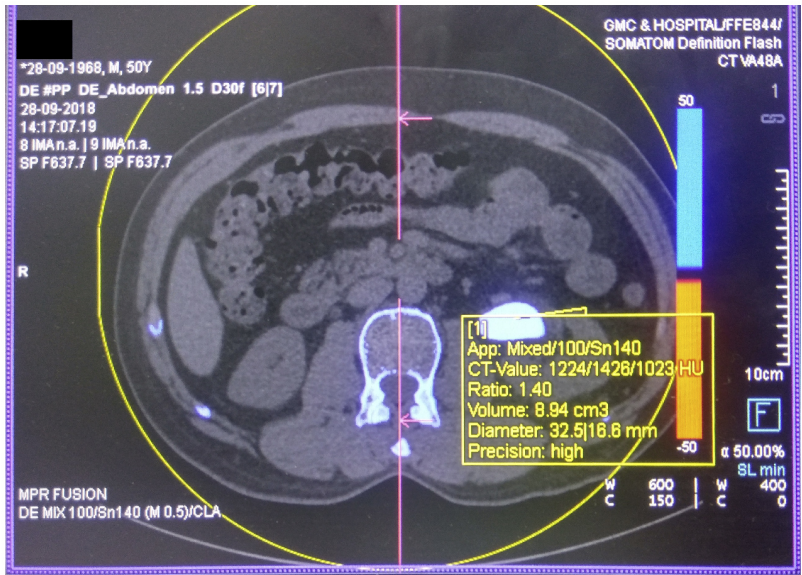

$\mathrm{D}$

Figure 5. Post-processed DECT images showing characteristics of calculi (colour, HU values at mixed/100/140 kVp and the DE ratio).

- (A) post-processed DECT showing blue coloured calculus with the DE ratio of 1.43 in the right inferior calyx corresponding to calcium oxalate calculus;

- (B) blue coloured calculus with the DE ratio of 1.40 in the left renal pelvis corresponding to calcium oxalate calculus;

- (C) calculus in the right upper ureter (red colour on colour map) with the DE ratio of 1.08 corresponding to uric acid calculus;

- (D) post-processed DECT showing blue coloured calculus with the DE ratio of 1.40 in the left renal pelvis corresponding to calcium oxalate calculus.

calculi from non- calcium oxalate calculi $(\mathrm{p}<0.001$ and kappa value $=0.883$ ).

The DECT has been reported as having a near $100 \%$ sensitivity and specificity for characterizing the chemical composition of renal stones measuring more than $3 \mathrm{~mm}$ [12]. The chemical composition of urinary calculi is a significant factor in predicting the effectiveness of shockwave lithotripsy $[17,18]$. Previously, stone analysis required imaging, laboratory, and pathology examinations [19]. Uric acid stone composition is of particular interest to predict as these stones can be treated through alkalinization of the urine [20].
We used a fourth generation 256-slice dual-source dualenergy scanner (SOMATOM Definition Flash; Siemens, Forchheim, Germany). Compared to earlier generation scanners, the use of fourth generation DECT at 100 and $140 \mathrm{kVp}$ in our study improved the classification of urinary calculi due to its better spectral separation hardware and software [21].

The strength of the current study is its prospective design that removes the selection bias of a retrospective study. 


\section{Conclusions}

DECT is highly sensitive in the differentiation of calcium oxalate calculi from non-calcium oxalate calculi (sensitivity and specificity of $97.5 \%$ and $90.9 \%$, respectively) and uric acid calculi from non-uric acid calculi (sensitivity and specificity of $100 \%)$. DECT is just as sensitive and accurate as chemical analysis in distinguishing uric acid calculi from non-uric acid calculi; thus, DECT can replace chemical analysis as a means of differentiating between uric acid calculi from non-uric acid calculi in the future which will aid in choosing the appropriate management strategy as uric acid calculi are amenable to drug treatment, while most of non-uric acid calculi require surgical intervention.

\section{Limitations}

Current limitation of DECT includes lesser efficacy in characterizing small stones measuring $<3 \mathrm{~mm}$; however, it has been found that such stones tend to pass spontaneously in majority of cases [22]. Radiation exposure is a concern that can be limited with radiation protection strategies and focused scanning. Before DECT, it is recommended to start with a low-dose abdominal CT scan to locate the stone. Respiratory movements are another limitation that requires the patient's full co-operation during the procedure.

Apart from DECT, there isn't any other non-invasive modality which can provide the accurate chemical composition of renal calculi preoperatively.

\section{Ethical Statement}

Permission to conduct this study was provided by the institutional Ethical Committee of Government Medical College (Srinagar, Jammu and Kashmir, India) in September 2017. All procedures contributing to this work comply with the ethical standards of the relevant national and institutional guidelines and the Declaration of Helsinki.

\section{Informed Consent}

Informed written consent was obtained from all the participants in the study.

\section{Conflict of Interest}

The authors declare that no conflicts exist.

\section{Financial Disclosure}

The authors declared no financial support.

\section{References}

[1] Eliahou R, Hidas G, Duvdevani M, Sosna J. Determination of renal stone composition with dual-energy computed tomography: an emerging application. Seminars in Ultrasound, CT and MRI. 2010;31(4):315-320. Available from: https://doi.org/10.1053/j.sult.2010.05.002
[2] Rule AD, Lieske JC, Li X, Melton LJ, Krambeck AE, Bergstralh EJ. The ROKS nomogram for predicting a second symptomatic stone episode. Journal of the American Society of Nephrology. 2014;25(12):2878-2886. Available from: https://doi.org/10.1681/ASN.2013091011

[3] López M, Hoppe B. History, epidemiology and regional diversities of urolithiasis. Pediatric Nephrology. 2010;25(1):49-59. Available from: https://doi.org/10.1007/s00467-008-0960-5

[4] Liu Y, Chen Y, Liao B, Luo D, Wang K, Li H, et al. Epidemiology of urolithiasis in Asia. Asian Journal of Urology. 2018;5(4):205-214. Available from: https://doi.org/10.1016/j.ajur.2018.08.007

[5] Konjengbam H, Meitei SY. Association of kidney stone disease with dietary factors: a review. Anthropological Review. 2020;83(1):65-73. Available from: https://doi.org/10.2478/anre-2020-0005

[6] Thongprayoon C, Krambeck AE, Rule AD. Determining the true burden of kidney stone disease. Nature Reviews Nephrology. 2020;16(12):736-746. Available from: https://doi.org/10.1038/s41581-020-0320-7

[7] Hidas G, Eliahou R, Duvdevani M, Coulon P, Lemaitre L, Gofrit ON, et al. Determination of renal stone composition with dual-energy ct: in vivo analysis and comparison with X-ray diffraction. Radiology. 2010;257(2):394-401. Available from: https://doi.org/10.1148/radiol.10100249

[8] Tanaka M, Yokota E, Toyonaga Y, Shimizu F, Ishii Y, Fujime $M$, et al. Stone attenuation value and cross-sectional area on computed tomography predict the success of shock wave lithotripsy. Korean Journal of Urology. 2013;54(7):454. Available from: https://doi.org/10.4111/kju.2013.54.7.454

[9] Dawoud MM, Dewan KAAWA, Zaki SA, Sabae MAA-R. Role of dual energy computed tomography in management of different renal stones. The Egyptian Journal of Radiology and Nuclear Medicine. 2017;48(3):717-727. Available from: https://doi.org/10.1016/j.ejrnm.2017.03.020

[10] Williams JC, Saw KC, Paterson RF, Hatt EK, McAteer JA, Lingeman JE. Variability of renal stone fragility in shock wave lithotripsy. Urology. 2003;61(6):1092-1096. Available from: https://doi.org/10.1016/S00904295(03)00349-2

[11] Primak AN, Giraldo JCR, Eusemann CD, Schmidt B, Kantor B, Fletcher JG, et al. Dual-source dual-energy CT with additional tin filtration: dose and image quality evaluation in phantoms and in vivo. American Journal of Roentgenology. 2010;195(5):1164-1174. Available from: https://doi.org/10.2214/AJR.09.3956 
[12] Li Z-X, Jiao G-L, Zhou S-M, Cheng ZY, Bashir S, Zhou Y. Evaluation of the chemical composition of nephrolithiasis using dual-energy CT in Southern Chinese gout patients. BMC Nephrology. 2019;20(1). Available from: https://doi.org/10.1186/s12882-019-1441-8

[13] Basha MAA, AlAzzazy MZ, Enaba MM. Diagnostic validity of dual-energy CT in determination of urolithiasis chemical composition: In vivo analysis. The Egyptian Journal of Radiology and Nuclear Medicine. 2018;49(2):499-508. Available from: https://doi.org/10.1016/j.ejrnm.2017.12.018

[14] Ilyas M, Dev G, Gupta A, Bhat T, Sharma S. Dualenergy computed tomography: A reliable and established tool for in vivo differentiation of uric acid from nonuric acid renal Stones. Nigerian Postgraduate Medical Journal. 2018;25(1):52. Available from: https://doi.org/10.4103/npmj.npmj_24_18

[15] Stolzmann P, Kozomara M, Chuck N, Müntener M, Leschka $S$, Scheffel $H$, et al. In vivo identification of uric acid stones with dual-energy CT: diagnostic performance evaluation in patients. Abdominal Imaging. 2009;35(5):629-635. Available from: https://doi.org/10.1007/s00261-009-9569-9

[16] Ahn SH, Oh TH, Seo IY. Can a dual-energy computed tomography predict unsuitable stone components for extracorporeal shock wave lithotripsy? Korean Journal of Urology. 2015;56(9):644. Available from: https://doi.org/10.4111/kju.2015.56.9.644

[17] Ferrandino MN, Pierre SA, Simmons WN, Paulson EK, Albala DM, Preminger GM. First prize (Tie): dual-energy computed tomography with advanced postimage acquisition data processing: improved determination of urinary stone composition. Journal of Endourology. 2010;24(3):347-354. Available from: https://doi.org/10.1089/end.2009.0193
[18] Stolzmann P, Scheffel H, Rentsch K, Schertler T, Frauenfelder T, Leschka S, et al. Dual-energy computed tomography for the differentiation of uric acid stones: ex vivo performance evaluation. Urological Research. 2008;36(3-4):133-138. Available from: https://doi.org/10.1007/s00240-008-0140-X

[19] Hartman R, Kawashima A, Takahashi N, Silva A, Vrtiska T, Leng S, et al. Applications of dual-energy CT in urologic imaging: an update. Radiologic Clinics of North America. 2012;50(2):191-205. Available from: https://doi.org/10.1016/j.rcl.2012.02.007

[20] Cheng PM, Moin P, Dunn MD, Boswell WD, Duddalwar VA. What the radiologist needs to know about urolithiasis: part 2??? CT findings, reporting, and treatment. American Journal of Roentgenology. 2012;198(6):W548-W554. Available from: https://doi.org/10.2214/AJR.11.8462

[21] Duan X, Li Z, Yu L, Leng S, Halaweish AF, Fletcher JG, et al. Characterization of urinary stone composition by use of third-generation dual-source dual-energy CT with increased spectral separation. American Journal of Roentgenology. 2015;205(6):1203-1207. Available from: https://doi.org/10.2214/AJR.15.14348

[22] Graser A, Johnson TRC, Bader M, Staehler M, Haseke $\mathrm{N}$, Nikolaou K, et al. Dual energy CT characterization of urinary calculi: initial in vitro and clinical experience. Investigative Radiology. 2008;43(2):112-119. Available from: https://doi.org/10.1097/RLI.0b013e318157a144

Received: 2021-06-09

Revised: 2021-07-25

Accepted: 2021-07-31 